\title{
Acuerdo de París: ¿nuevos compromisos con el medio ambiente o nuevas oportunidades de negocio?
}

\author{
Simone Di Pietro**
}

\footnotetext{
Artículo de reflexión

*Politólogo de la Pontificia Universidad Católica del Ecuador. Docente de Nuevos compromisos globales con el Medio Ambiente. Magíster en Energías Renovables en la Universidad Politécnica de Cartagena de España, y Magíster en Ciencias Políticas en la Universidad Sapienza de Roma, Italia. Correo electrónico: simonedipietrosapienza@gmail.com
}

Recibido: 29 de agosto del 2016 Aprobado: 22 de enero del 2017

Cómo citar este artículo: Di Pietro, S. (2017). Acuerdo de París: ¿nuevos compromisos con el Medio Ambiente o nuevas oportunidades de negocio? Cooperativismo \& Desarrollo, 111(25), 43-51. doi: https://doi.org/10.16925/ co.v25i111.1874

\section{Resumen}

Propósito: el presente artículo es resultado de un proceso de investigación llevado a cabo en el marco del curso "COP21: Nuevos compromisos con el Medio Ambiente", dictado en el 2016 en la Universidad Católica del Ecuador. Su propósito es realizar una revisión del Acuerdo de Paris, adoptado en noviembre del 2015, por parte de los países constituyentes de la vigésimo primera conferencia de la Convención Marco de las Naciones Unidas sobre el Cambio Climático (CMNUCC). Temas: después de un análisis sobre las evidencias del cambio climático y sus efectos, se analizan las diferentes políticas productivas y económicas propuestas internacionalmente como soluciones viables respecto a los cambios presentes y futuros, tanto a nivel social, como productivo. Desarrollo: en la segunda parte del artículo se revisa la historia colonial con matriz imperialista, y se deja al lector con la pregunta abierta, de si las nuevas soluciones propuestas tienen efectivamente el potencial para mitigar el cambio climático, o si más bien son parte de un neocolonialismo económico, resultado de la capacidad de readaptación constante del capitalismo. Conclusiones: por fin, se deja un espacio de discusión abierto sobre algunas propuestas interesantes, de inspiración latinoamericana, como el posdesarrollismo y el poscapitalismo.

Palabras clave: Acuerdo de París, imperialismo económico, posdesarrollismo. 


\title{
Paris Agreement: New environment commitments or new business opportunities?
}

\begin{abstract}
Purpose: This article is the result of a research process carried out within the framework of the course "COP21: New Commitments with the Environment", imparted in 2016 at the Universidad Católica del Ecuador. Its purpose is to carry out a review of the Paris Agreement, adopted in November 2015, by the constituent countries of the twenty-first conference of the United Nations Framework Convention on Climate Change (UNFCCC). Themes: After analyzing the evidence of climate change and its effects, further evaluation is done regarding the different productive and economic policies proposed internationally as viable solutions to current and future changes, both socially and productively. Development: In the second part of the article, colonial history with an imperialist matrix is reviewed, and the reader is left with an open question: whether the new solutions proposed effectively have the potential to mitigate climate change, or if they are actually part of an economic neocolonialism, resulting from capitalism's capacity for constant readjustment. Conclusions: Finally, an open discussion space is offered on some Latin American interesting proposals, such as post-development and post-capitalism.
\end{abstract}

Keywords: Paris Agreement, economic imperialism, post-development

\section{Acordo de Paris: novos compromissos com o meio ambiente ou novas oportunidades de negócio?}

\section{Resumo}

Propósito: este artigo é resultado de um processo de pesquisa realizado no âmbito do curso "COP21: novos compromissos com o meio ambiente", ministrado em 2016 na Universidad Católica del Ecuador. Seu propósito é realizar uma revisão do Acordo de Paris, adotado em novembro de 2015 por parte dos países constituintes da vigésima primeira conferência da Convenção-Quadro das Nações Unidas sobre Mudança do Clima (UNFCCC, por sua sigla em inglês). Temas: após analisar as evidências da mudança climática e de seus efeitos, avaliam-se as diferentes políticas produtivas e econômicas propostas internacionalmente como soluções viáveis a respeito das mudanças presentes e futuras, tanto no âmbito social quanto no produtivo. Desenvolvimento: na segunda parte do artigo, revisa-se a história colonial com matriz imperialista e deixa-se a pergunta aberta sobre se as novas soluções propostas têm efetivamente o potencial para mitigar a mudança climática ou se são parte de um neocolonialismo econômico, resultado da capacidade de readaptação constante do capitalismo. Conclusões: por fim, deixa-se um espaço de discussão sobre algumas propostas relevantes, de inspiração latino-americana, como o pós-desenvolvimentismo e o pós-capitalismo.

Palavras-chave: Acordo de Paris, imperialismo econômico, pós-desenvolvimentismo. 


\section{Introducción}

El presente artículo busca ofrecer una visión crítica acerca de las políticas de economía ecológica, presentadas por las instituciones globales en respuesta a los problemas relacionados de forma directa o indirecta con el cambio climático, a partir de la última década del siglo xx. Esto con el fin de poner en evidencia cómo en muchos casos estas políticas tienen un carácter hegemónico y mercantil incapaz de crear los cambios sociales estructurales necesarios para revertir eficazmente el fenómeno del cambio climático. Se pretende poner en evidencia el carácter una vez más insuficiente de los acuerdos logrados, en relación con sus probabilidades de alcanzar los resultados esperados, en conformidad con la falta de éxito de los acuerdos precedentes.

El Acuerdo de París, adoptado el día 12 de diciembre del 2015 al término de la COP21, establece que el aumento de la temperatura global no debe sobrepasar el nivel límite de $1,5^{\circ} \mathrm{C}$ respecto a los niveles preindustriales. Para entrar en vigor en el 2020, es necesario que obtenga la ratificación y aprobación de los parlamentos de por lo menos los 55 países que juntos representan el $55 \%$ de las emisiones globales de GEI. La Alianza de los Pequeños Estados Insulares, como países más expuestos al incremento del nivel del mar, ya se apresuró a ratificar el acuerdo y ahora piden al resto del mundo no perder tiempo.

Autores como Hansen et al. calculan que existe un desfase entre los cambios en los forzamientos climáticos y la respuesta del clima (Ballester, Díaz y Moreno, 2006, p. 161). Según los cambios térmicos observados y la energía acumulada en el sistema climático, estos autores calculan que hay energía almacenada en el planeta para asegurar un calentamiento adicional de $0,6^{\circ} \mathrm{C}$. En otras palabras, el calentamiento del planeta no es de mañana, es de ayer, y continuará hasta por lo menos $1,4^{\circ} \mathrm{C}$, suponiendo que volviésemos inmediatamente a la situación de hace 200 años. En este contexto, resulta difícil pensar realizable el objetivo de no sobrepasar el nivel límite de $1,5{ }^{\circ} \mathrm{C}$, tal como plantea el Acuerdo de París.

Según Brand, en un sistema que se basa en la maximización de la ganancia, simplemente no es posible obtener crecimiento de la economía y la protección del medio ambiente al mismo tiempo (Peters 2014, p. 130).

Keynes, en 1930, mencionaba que el límite absoluto de saturación en términos de consumo llegaría en el 2030 (Acosta 2014, p. 102). Parece que su previsión se esté revelando exacta, pues a pesar de esto las grandes instituciones internacionales están basando los acuerdos climáticos sobre políticas de adaptación y mitigación del cambio climático, sin plantear cambios en el paradigma del sistema económico dominante.

\section{Estrategias de desarrollo en el marco de la globalización}

Los acuerdos de reducción de emisiones y los mecanismos introducidos por el Protocolo de Kioto, más allá de haber dado vida a algunos efectos perversos, se han demostrado ampliamente insuficientes, así como el nuevo acuerdo de París que no prevé ningún mecanismo de sanción legal para los países que no cumplen con sus compromisos de reducción de emisiones "voluntarios", o parece por lo menos dudoso. La Low Emission Development Strategy, o "Estrategia de Desarrollo bajo en Emisiones", como serie de políticas promovidas por la CMNUCC con el objetivo de disociar el crecimiento económico de las emisiones de GEI en todo el mundo, corre el riesgo de convertirse en términos trágicos, en la "utopía del siglo xxı".

El pensamiento de la OECD (2008) se puede resumir en pocas palabras extraídas de su informe "Perspectivas medioambientales para 2030":

La liberalización del comercio y de la inversión puede fomentar una asignación de recursos más eficiente a nivel global si se cuenta con una política ambiental y un marco institucional acertados (...) Asegurarse que la globalización lleve a un uso más eficiente de los recursos y al desarrollo y difusión de la eco innovación (...) Soluciones tecnológicas están ofreciendo nuevas perspectivas de desarrollo como por ejemplo la cCs (Carbon Capture and Storage) y vehículos híbridos que se pueden volver cada vez más competitivos en términos de coste o el aprovechamiento de la biomasa de los residuos que permitirá limitar la expansión de tierras agrícolas para la producción de biocombustible.

La captura y el almacenamiento del carbono, muy exaltadas por las instituciones por su posibilidad de reducir la presencia de carbono en la atmosfera o por sus posibilidades de negocio, implican procesos químicos que producen la separación y el adelgazamiento del carbono.

Desde una perspectiva optimista sería posible reducir entre el 80 y $90 \%$ de $\mathrm{CO} 2$ generado por las centrales termoeléctricas, pero en este caso los costes de producción de electricidad aumentarían entre 35 y $85 \%$, además de los problemas de almacenamiento 
seguro del carbono aislado (IPCC, 2005). Entre una de las ideas propuestas, el almacenamiento oceánico parece tener muy poco sentido, considerando los problemas actuales de acidificación de los océanos, debido a que los mares absorbieron el 60 \% del dióxido de carbono, emitido de forma antropogénica desde la Revolución industrial.

Por cierto, la implementación de estas tecnologías, aunque perfeccionadas, llevarían a un aumento importante de los costes de producción de la energía, así como las propuestas de introducir impuestos fiscales sobre las externalidades negativas industriales representadas por las emisiones de GEI. Todo esto podría terminar favoreciendo la expansión de los biocombustibles en el mercado de la energía.

A nivel global, las energías renovables están en competición con los combustibles fósiles en el mercado de la energía. El petróleo hasta el momento sigue teniendo la mejor tasa de retorno energético (Villada, 2016), mientras que entre los biocombustibles se encuentra el etanol de la caña de azúcar, el biodiesel de la soja y el etanol del maíz, cuyos cultivos han sido subvencionados especialmente en el Cornvalley de Estados Unidos desde la época Bush, también a pesar de su escaso potencial energético, a fin de favorecer intereses de los lobbies típicos del sistema político estadounidense.

El aprovechamiento de la energía solar tiene la cualidad de ser un sistema de producción de energía bastante limpio. Los paneles fotovoltaicos están constituidos de silicio, un elemento muy abundante en la naturaleza. Los inconvenientes surgen en la relación entre el potencial energético y la superficie necesaria: se estima que aprovechando todo el potencial fotovoltaico de la tierra, o sea cubriendo cada metro cuadrado de la superficie terrestre, apenas alcanzaríamos a satisfacer la demanda energética mundial del 2015 (REN21, 2015, p. 59).

Las opciones más eficientes en cuanto a tasa de retorno energético, entre las energías alternativas actualmente resultan ser la energía hidroeléctrica y eólica, aunque estas también impactan negativamente sobre la fauna de los ríos, la fauna avícola, además de afectar el curso de los ríos y provocar, en ciertos casos, deforestación.

Por cierto, la instalación de pequeños sistemas de EERR resulta óptima para la seguridad energética de áreas rurales. Respecto a la implementación de EERR en sistemas de red eléctrica nacional, desde el punto de vista legislativo, lo más natural sería permitir las políticas de autoconsumo con balance neto, las cuales permiten a los consumidores generar su propia electricidad a partir de fuentes de EERR y contribuir con una inyección extra de energía a la red general, ya sea para percibir una remuneración con base en los términos contractuales en vigor. También en este caso, la lógica del capital corresponde a un límite para la sociedad con buenas intenciones: no se explica de otra manera la prohibición de estas políticas en España, sino como medida para imponer el oligopolio de las empresas eléctricas en el país. Sobre este tema cabe mencionar el trabajo de la Fundación Desarrollo Sostenible, la cual, desde hace algunos años trabaja en la campaña nacional " Corta los cables! La autosuficiencia eléctrica es posible", con el propósito de invitar a los usuarios de la energía a instalar su propios sistemas de autoconsumo independientes de la red nacional, como respuesta de soberanía energética y empoderamiento ciudadano. ${ }^{1}$

Es probable que la cuestión de la tierra constituya el problema más importante para las generaciones futuras, si no se cambia la legislación acerca de su uso y propiedad.

La OECD estima que a los ritmos de crecimiento actuales, en el 2050 la producción combinada de alimentos y biocombustible, exigirá un $10 \%$ de incremento de tierra de labor en todo el mundo, con la predominancia del modelo de cultivo industrial. Actualmente, el $50 \%$ de los cereales se utilizan para el consumo animal y agrocombustibles. El monocultivo y el tipo de producción industrial con elevado uso de fertilizantes y pesticidas, produce serios daños a la biodiversidad y al medio ambiente, en general. Además, es necesario evidenciar cómo, mientras el bosque húmedo tropical en su estado nativo almacena alrededor de $200 \mathrm{t}$ de carbono por hectárea, un bosque degradado o intervenido almacena $70 \mathrm{t}$ de carbono; un área cultivada, dependiendo del tipo de cultivo, puede tener hasta $45 \mathrm{t}$, mientras que las áreas de pasto generalmente contienen solo 2,5 t por hectárea. De esta manera, cada vez que se afecta la naturaleza en su estado nativo, vamos perdiendo un potencial importante para mitigar el calentamiento global.

Uno de los fenómenos más importantes del siglo XxI es sin duda el acaparamiento de tierras, o land grabbing en inglés. Los inversores de todo el mundo se han dado cuenta que el precio de la tierra sigue aumentando en todo el mundo, y cómo la tierra fértil se ha convertido en una inversión relativamente segura en todos los rincones del planeta.

Véase al respecto http://www.cortaloscables.com/por-que-esta-iniciativa/ 
Se calcula que en los últimos años han cambiado de manos al menos 227 millones de hectáreas, la mayor parte en África subsahariana (White, 2012). Como evidencia Stefano Liberti (2014) en Nuevos Amos de la Tierra, a raíz de este nuevo fenómeno se desata la crisis financiera del 2008 en los países enriquecidos, cuyos actores buscan nuevos activos seguros que encuentran en los mercados de materias primas alimenticias; además, la crisis energética que despierta el interés para la producción de biocombustible y el constante crecimiento demográfico destinado a no parar.

Es así que, en nombre del desarrollo, aunque a través de acuerdos envueltos en el secretismo, los gobiernos de países emergentes o empobrecidos, en connivencia con el Banco Mundial y el Fondo Monetario Internacional, apoyan las transacciones de vastas extensiones de tierra a grandes corporaciones, tanto nacionales, como extranjeras, tratándose habitualmente de concesiones o arrendamientos a largo plazo (99 años), más que de compras (White, 2012).

En muchos casos se registra la expulsión o afectación de comunidades enteras de los espacios en los que vivían, ya que en estos países suelen no existir títulos sobre la tierra, sino que se trata de derechos consuetudinarios o comunales de propiedad estatal.

Barari Nyari, una ONG ghanesa, describe cómo la empresa noruega de agrocombustibles Agrofuel África, aprovechó el sistema tradicional de tenencia de la tierra comunitario en el norte de Ghana para apropiarse y deforestar grandes extensiones de tierra y crear la plantación de jatropha más grande del mundo. Persuadieron a la población local analfabeta para firmar contratos con una simple huella dactilar, alimentando expectativas de empleo e ingresos que no se materializaron a medida que los bosques se iban vaciando y la población perdía los ingresos de sus productos forestales (White 2012, p. 11).

Entre los acaparadores de tierra, cabe señalar a China, Arabia Saudí, otros países del golfo pérsico y otros países ricos entre los que se destaca el Reino Unido. En particular, Arabia Saudí decidió, en el 2008, mejorar su seguridad alimentaria invirtiendo a través de su Fondo Soberano de Inversión Abu Dhabi Investment Authority, en arrendar y producir alimentos para importación en las llanuras fértiles de Etiopía.

Así, mientras en África, que es el continente más vulnerable y expuesto a los efectos del cambio climático, se invierte en producción de alimentos para exportación y biocombustibles, explotando mano de obra barata y bajos costes de producción, el Mecanismo
Desarrollo Limpio introducido en Kyoto evidencia una distribución desigual de los proyectos: solo el 2,9 \% se localiza en África, mientras que Asia y el Pacífico representan más del $80 \% .{ }^{2}$ Mientras tanto, los Fondos Soberanos de Inversión, que son vehículos de inversión de propiedad estatal, gestionados por los bancos centrales y cuyos capitales generalmente provienen de la exportación de materias primas como gas o petróleo, invierten principalmente en países desarrollados como Reino Unido, EEUU o España, en los sectores tecnológicos, banqueros, edilicios o de la energía, llegando en ciertos casos a ganar el control en sectores estratégicos de los países receptores. Por ejemplo, el gobernador de China, Development Bank, toma parte del consejo directivo del banco británico Barclays.

El periodista británico Sebastian Mallaby consideró que las protestas del senador norteamericano Jim Webb sobre la compra de Blackstone por China, se debieron a que China puede llegar a ser propietaria de información sensible (Santiso, 2014).

\section{Violencia y capitalismo, colonialismo y extractivismo}

El colonialismo fue una verdadera doctrina del desarrollo. El capitalismo tiene la necesidad constante de expandirse y es el factor económico de raíz de casi todos los conflictos de la historia. Así fue que, como consecuencia de la Revolución industrial, Europa necesitaba expandirse en busca de regiones que proporcionaran materias primas y mercados:

El extractivismo es una modalidad de acumulación que comenzó a fugarse masivamente hace 500 años, constituye una categoría que permite explicar el saqueo, acumulación, concentración, destrucción, y devastación colonial y post-colonial así como la evolución del capitalismo hasta ahora. Con la conquista y colonización de AL, África y Asia comenzó a estructurarse la economía mundial como uno de los elementos fundacionales de la civilización capitalista se desarrolla y consolida la modalidad de acumulación extractiva determinada por los centros metropolitanos. (Acosta, 2014, p. 106)

Las burguesías presionaban a los gobiernos para invertir capitales en ferrocarriles, puertos, y realizar préstamos a autoridades locales. Además, las colonias

Véase al respecto Grupo de Alto Nivel para el Diálogo Político del MDL (2012); y wWF (2013). 
eran fuente de materias primas tales como oro, diamantes, algodón, seda o hevea. En la conferencia de Berlín de 1884-85, los países europeos se reparten África y la sumisión de su pueblo. Más allá de las razas étnicas, en 1890, al descubrirse diamantes y oro en las pequeñas repúblicas libres del Orange y Transvaal de origen boer-holandes, estalló la guerra de Boers en 1899 , en la que los ingleses se apoderaron de las dos repúblicas, y crearon en 1910 la Unión Sudafricana (Martín-Mateos, 2009).

Otra guerra colonial movida por el capitalismo fue la Guerra del Opio, la cual comenzó en 1839 cuando las autoridades chinas destruyeron un cargamento de opio en Cantón. El emperador se había dado cuenta de cómo los intereses de la East India Company británica en el comercio del opio estaban envenenando su pueblo. Los británicos respondieron a esta acción enviando buques de guerra en 1840 . Obtuvieron una rápida victoria tras la firma del tratado de Nanjing, con lo que China se comprometía a pagar una gran indemnización, abrir sus puertas al comercio exterior y ceder Hong Kong al Reino Unido.

En África los procesos de independencia fueron fomentados por la onu desde 1945, con base en el principio de autodeterminación de los pueblos contenido en su carta. Los procesos independentistas se cumplieron entre la década de los sesenta y la de los ochenta, acompañados por nuevas ideologías de panafricanismo y negritud, concebidas y promovidas a nivel intelectual por personajes de la elite colonial africana que habían asimilado ideales independentistas al entrar en contacto, en Europa, con los ambientes intelectuales y universitarios, como, por ejemplo, Frantz Fanón y Du Bois.

Lejos de una emancipación real, África se convirtió, durante la guerra fría, en uno de los escenarios principales en los que se enfrentaron Estados Unidos y la Unión Soviética. Angola, por ejemplo, sufrió tras su independencia, conseguida en 1975, después de 14 años de enfrentamientos contra Portugal, el inicio de una guerra configurada por dos partes: el movimiento para la liberación de Angola (MPLA) de José Eduardo Dos Santos, quien obtuvo el apoyo incondicional de la Unión Soviética y de 60000 soldados cubanos. Por otra parte, la Unión Nacional para la Independencia total de Angola (UNITA), liderada por Jonas Savimbi, fue respaldada directamente por EEUU y por el gobierno sudafricano del Apartheid. En el 2002, la muerte del líder guerrillero Jonas Savimbi llevó al acuerdo de paz, y dejó un reguero de un millón de muertos y 1/10 parte de la población mutilada por minas. Este conflicto se sustentaba por la venta del petróleo por parte del gobierno Dos Santos a empresas como Chevron, Elf, Aquitaine, BP, Exxon, y la de los diamantes por parte de la unita (Martín-Mateos, 2009).

Al final de la guerra fría muchos países africanos iniciaron una fase de cambios profundos fundamentada en el fracaso de la consolidación del Estado nacional. Más de 30 países africanos se vieron inmersos en una ola democratizadora que trascurrió de forma pacífica: Benín, Cabo Verde, Malawi, Mali, Mozambique, Namibia, Níger, Santo Tomé y Príncipe, Lesotho, República Centroafricana, Congo, Guinea Bissau, Sudáfrica y Zambia. En otro grupo de países la respuesta al proceso de erosión estatal desembocó en el derrumbamiento del Estado poscolonial, y el inicio de conflictos bélicos sangrientos como los de Angola, Burundi, Chad, Liberia, República Democrática del Congo, Ruanda, Sierra Leona, Somalia y Sudan.

Algunos factores llevaron al debilitamiento y la erosión de los Estados poscoloniales: los líderes de la independencia prefirieron dar continuidad a las estructuras político-administrativas y económicas que el colonialismo había impuesto, en lugar de sustituirlas con estructuras políticas autóctonas. Así se conservaron unas instituciones caracterizadas por fronteras artificiales y estructuras administrativas diseñadas para explotar las divisiones locales y satisfacer las necesidades de las metrópolis, gracias a la exportación de productos agrícolas, minerales y materias primas. La naturaleza personalista y patrimonial de las elites africanas que centralizaron el poder político y económico al suprimir el pluralismo político y concentrar los recursos en sus comunidades étnicas de origen, perdieron el apoyo y la legitimidad del resto de los grupos étnicos. También participaron, desde el punto de vista económico, los efectos de una década de ajustes estructurales de las economías con base en las condiciones de préstamos impuestos por el Banco Mundial y el Fondo Monetario Internacional, según la lógica del Consenso de Washington, junto con la retirada del respaldo bilateral que desembocó en el colapso estatal en países como Liberia y Somalia.

Un escenario característico de la violencia en África causada por el control de las materias primas es Nigeria. En la región del Delta del Níger, que concentra el $60 \%$ del petróleo de Nigeria, país primer productor de crudo de la región subsahariana, varias milicias armadas pertenecientes a diferentes grupos étnicos se enfrentan entre ellas y contra las fuerzas de seguridad estatal por el control del poder y los beneficios del petróleo. También participan ejércitos 
privados contratados por las transnacionales del petróleo tales como Total, Elf y Shell. En la década de 1990, el Mouvement for the Survival of the Ogoni People (MOSOP), una población originaria de la región presentaron el "Ogoni Bill of Rights" al gobierno militar de Babangida, por el cual pedían autonomía política. La protesta se direccionaba contra la Shell, responsable de la destrucción del medio ambiente. El Mosop denunció al gobierno nigeriano y a la Shell frente al subcomité de los derechos humanos de la onu, y lanzó un ultimátum a Shell y Chevron, amenazando con la destrucción de los pozos. Fue entonces que el gobierno envió el ejército a proteger las instalaciones. Después de varios enfrentamientos, el líder del mosop, Ken Saro Wiwa, fue apresado y condenado a muerte en 1995.

África, como mercado para la venta de las armas producida en los países desarrollados, es seguramente un elemento estratégico sobre el que se basa el sistema capitalista en la época de la globalización. El financiamiento militar en el 2015 es 15 veces mayor que la ayuda al desarrollo.

Según el Informe del 2014 del Instituto Internacional para la $\mathrm{Paz}$ de Estocolmo, el gasto de eEuu fue de us 610000 millones (3,5\% del PIB); de Pekín, de us 216000 millones (2,1\% del PIB); y Moscú 84500 (4,5\% PIB).

La actitud bélica de los hombres no es nueva. Antiguamente, las consecuencias ambientales de las actividades militares eran mínimas y, en el peor de los casos, se limitaba al incendio de los bosques o el envenenamiento de víveres, lo que producía efectos limitados en el tiempo y el espacio. En guerras más recientes se han desplegado nuevos tipos de armas tales como municiones de alto poder explosivo, agentes químicos y materiales incendiarios, lo cual produce efectos ambientales mayores.

La Segunda Guerra Mundial, por ejemplo, causó una reducción de la productividad agraria a corto plazo del $38 \%$ en 10 naciones (Portilla, 1986, p.13).

En la guerra del Vietnam, los herbicidas químicos que el gobierno norteamericano compraba a empresas como Monsanto, destruyeron por completo $15000 \mathrm{~km}^{2}$ de manglares. El denominado "agente naranja" contenía como impureza más de $100 \mathrm{~kg}$ de dioxina, y la difusión de esta sustancia ha causado, desde entonces, defectos en los nacimientos, abortos, y cáncer de hígado en los seres humanos.

Otro herbicida como el glifosato fabricado por Monsanto, ha sido utilizado en la última década para las fumigaciones de los campos de coca en el Plan
Colombia, gestionado por los gobiernos colombiano y estadunidense para combatir el cultivo ilícito, enriqueciendo así la ya poderosa multinacional norteamericana y reduciendo a la pobreza extrema a los campesinos colombianos.

Como afirma Peters (2014), el mundo desarrollado se encuentra en una crisis profunda y estructural. El crecimiento económico está estancado y países de la periferia europea atraviesan una fuerte y prolongada recesión. La crisis económica se transformó en una crisis social debido al aumento de las tasas de pobreza, el desempleo y las desigualdades sociales en los países del norte. El malestar generalizado se convierte en reivindicaciones de democracia directa y los movimientos de ultraderecha van ganando terreno. Así, se explica la avanzada electoral de movimientos políticos en Europa como Podemos, en España, Movimento 5 Stelle, en Italia, Front National, en Francia, Syriza, en Grecia, y la extrema derecha del Partido de la Libertad Austriaco, en Austria, que están metiendo en crisis los sistemas bipartidistas.

\section{Conclusiones}

Es evidente, una vez más, la presencia, dentro de los acuerdos de París, de la lógica ortodoxa capitalista en la economía globalizada actual. La experiencia de los Acuerdos del Protocolo de Kioto, en vigor desde el 2005 , nos enseña que es imposible llegar a los resultados esperados en materia de cambio climático dentro de este paradigma que por medio de los Estados, instrumentaliza las sociedades hacia la maximización de la ganancia.

Según el Informe de los Objetivos del Milenio de 2015, desde 1990 al 2015 se han logrado progresos relevantes en la lucha contra la pobreza. Carlos Larrea (2014) evidencia las tres dimensiones insatisfactorias del crecimiento económico a nivel global: el crecimiento económico ha sido acompañado por aumento de la desigualdad social, conduciendo al mantenimiento del $50 \%$ de la población de los países en vía de desarrollo bajo la línea de la pobreza, mientras los beneficios se han concentrado crecientemente en una fracción reducida de la población. En el 2010, mientras el $10 \%$ más rico de la población mundial continuaba en condiciones de pobreza con un ingreso por habitante inferior a us 2,5 por día, el ingreso medio anual de us 8,25 diarios por persona duplica la línea de vulnerabilidad, y es aproximadamente cuatro veces mayor a la línea de la pobreza. Las otras dos dimensiones insatisfactorias del crecimiento 
son la no sustentabilidad del crecimiento por parte del planeta, y que la asociación entre la realización humana y la mayor posesión de bienes y servicios, más allá de la satisfacción de necesidades básicas, se ha mostrado débil. Según Larrea, las únicas opciones viables son la reducción del tamaño de las economías hasta límites sustentables, o la reducción de su impacto ambiental.

Respecto a la reducción del impacto ambiental, para un contexto generalizado de despliegue mundial de tecnologías limpias capaces de facilitar energía y productos para los niveles de consumo crecientes, garantizando al mismo tiempo el mínimo impacto ambiental sustentable por el planeta, será necesario esperar la implementación de nuevos inventos más eficientes, limpios y capaces al mismo tiempo de abastecer la demanda mundial. Hasta alcanzar este momento, será necesario reorganizarse con base en alternativas sociales que cuestionen el desarrollo e interpretación dominante del crecimiento económico. Acosta (2014) habla de inmediato de decrecimiento planificado del extractivismo y la superación del concepto mismo de desarrollo, dando paso a alternativas como el buen vivir, a través de un proceso que pase por la desmercantilización de la naturaleza, la reorganización de la producción desenganchada de los engranajes de los mecanismo actuales de mercado y el principio de descentralización en temas de seguridad alimentaria y energética. Hay que dar paso a una transformación histórica y una concepción biocéntrica desde una antropocéntrica.

Un tema a tener en cuenta es que la mayoría de la población condenada sistemáticamente a la exclusión y a la pobreza no reflexiona estas cuestiones, y aspira permanentemente a vivir con los niveles de consumo de los grupos más acomodados. Así, se justifican los resultados electorales de los últimos años en Bolivia y Ecuador, países donde la introducción constitucional del buen vivir con su potencial revolucionario, ha cedido el paso a políticas neoextractivas para fortalecer el Estado hasta el último rincón, construir patrias.

Las concepciones de buen vivir y plurinacionalidad, como autodeterminación política, autonomía de las comunidades y, sobretodo, la búsqueda de construir una sociedad alternativa, se han quedado en la literatura, mientras después de una década de bonanza económica debida a los favorables precios internacionales de materias primas, Bolivia y Ecuador se encuentran con las inquietudes de una soberanía económica inestable, frente al riesgo de ver caer como un castillo de cartas los proyectos político avanzados en los últimos años por el MAs en Bolivia, y por Alianza País en Ecuador.

Desde una antropología que se debe a los pueblos indígenas amazónicos, encontramos el registro de una ontología social y cosmopolita contra el Estado y contra toda la globalización unificadora. Estas imágenes luchan contra el etnocentrismo productivista e individualista occidental, y presentan las opciones de sociedades tribales o nómadas no como desarrollo, sino como opción positiva existencial. El antropólogo brasileño Viveiros de Castro destaca la descripción de la sociedad indígena como máquinas antiproducción, en tensión con todo economicismo, bien sea liberal, marxista, o bien andino céntrico (Schavelzon, 2015, p. 26).

Día a día, en América Latina se observa una búsqueda de autonomía, plurinacionalidad y buen vivir. El pueblo Sarayaku, las comunidades del Tipnis boliviano, o de Junín en Intag y MahulloKhota, en Potosí, usan estrategias de movilización, marchas nacionales, expulsión de invasores desarrollistas o resistencias subterráneas. En este contexto se enmarcan las experiencias de gobierno autónomo recreando instituciones propias como, por ejemplo, la Alcaldía de Cotacachi en Ecuador, o los 11 primeros municipios autónomos en Bolivia, así como los 156 Suyus ancestrales reconstituidos en la Asamblea del Pueblo Guaraní que construye su autonomía.

Para el buen vivir, la riqueza no consiste en acumular la mayor cantidad posible de bienes, sino en lograr un equilibrio entre las necesidades fundamentales de la humanidad y los recursos disponibles para satisfacerla. Todo esto se enfrenta a aquellas visiones de desarrollo sostenible que era, y continua siendo, enarbolada por las empresas que quieren convertir el llamado "desarrollo verde y ecológico" en una nueva oportunidad de negocio. Según Acosta (2014), el posdesarrollo concibe que las personas y las comunidades no están necesariamente abocadas a satisfacer sus necesidades materiales, pues ellas forman parte de una constelación más amplia de necesidades construidas culturalmente. Justo estas construcciones culturales representan el límite social más grande respecto a la posibilidad de una reorganización posdesarrollo, constituyendo una barrera a la toma de conciencia ciudadana.

En conclusión, se evidencia que los acuerdos de París se caracterizan por una lógica economicista y productivista que no se aleja del pasado. Se trata, así, de hacer pasar el capitalismo con una nueva cara de salvador del mundo, gracias a los inventos y beneficios 
que derivará de nuevas inversiones, en muchos casos transferencias públicas hacia empresas privadas, mientras estas acciones por su debilidad y con carácter más reformista que revolucionario, difícilmente llegarán a conseguir los resultados esperados. Con toda probabilidad, la falta de determinación de hoy será la pesadilla del mundo de mañana. Al analizar la historia, nos damos cuenta de cómo, en la mayoría de los casos, los cambios estructurales de las sociedades surgen desde la base de la pirámide social. Así es que la reorganización desde un pueblo que tenga como prioridad absoluta garantizar su soberanía alimentaria y energética, es una característica presente siempre y que siempre volverá en las sociedades de cada época.

\section{Referencias}

Acosta, A. (2014). Post-crecimiento y post-extractivismo: dos caras de la misma transformación cultural. Quito: Friedrich Ebert Stiftung Ecuador FES-ILDIS.

Amnesty International. (2009). Petrolio, inquinamento e povertà nel Delta del Niger. Recuperado de https://clusonein3d.files.wordpress.com/2016/04/ nigeria.pdf

Ballester, F., Díaz F. J., y Moreno, J. M. (2006). Cambio climático y salud pública: escenarios después de la entrada en vigor del Protocolo de Kioto. Recuperado de http://www.sciencedirect.com/science/article/ pii/ S0213911106715801

Grupo de Alto Nivel para el Diálogo Político del MDL. (2012). Mercado de carbono y mecanismo desarrollo Limpio: llamado a la acción. Luxemburgo: CDM Policy Dialogue.

Hansen, J., Nazarenko, L., Ruedy, R., Sato, M., Willis, J, y del Genio, A., ... y y Tausnev, N. (2005). Earth's Energy Imbalance: Confirmation and Implications. Science. doi: https://doi.org/10.1126/science.1110252

IPCC Special Report. (2005). Carbon Dioxide Capture and Storage Technical Summary. Recuperado de https:// www.ipcc.ch/pdf/specialreports/srccs/srccs_ technicalsummary.pdf

Larrea, C. (2014). Límites de crecimiento y líneas de codicia: un camino hacia la equidad y sustentabilidad. Quito: Friedrich Ebert Stiftung Ecuador FES- ILDIS. Recuperado de http://library.fes.de/pdf-files/bueros/ quito/11348.pdf
Liberti, S. (2014). Nuevos Amos de la Tierra. Madrid: Taurus Pensamiento.

Martin-Mateos, O. (2009) África El continente maltratado. Guerra, expolio e intervención internacional en el África negra. Cuadernos de Cristianisme i Justicia. Cuadernos de Cristianisme i Justicia. Recuperado de https://www.cristianismeijusticia.net/sites/default/files/pdf/es137_0.pdf

Organización para la Cooperación y el Desarrollo Económicos-OECD. Prospectiva Medioambiental de la OECD para 2030. Recuperado de http://www.oecd. org/env/indicators-modelling-outlooks/40224072.pdf

Peters, S. (2014). Post-crecimiento y buen vivir: ¿discursos políticos alternativos o alternativas políticas. FES PostCrecimiento y Buen Vivir.

Portilla, J. M. (1986). Efectos ambientales del Armamentismo. Recuperado de http://bibliohistorico.juridicas.unam. $\mathrm{mx} / \mathrm{libros} / 1 / 249 / 17 . p d f$

REN21. (2015). Global Status Report. Recuperado de http://www.ren21.net/wp-content/uploads/2015/07/ REN12-GSR2015_Onlinebook_low1.pdf

Santiso, J. (2014). Fondos Soberanos 2014. Barcelona: ESADE Business School.

Schavelzon, S. (2015). Plurinacionalidad y Vivir Bien/Buen Vivir. Dos conceptos leídos desde Bolivia y Ecuador post-constituyentes. Clacso. Recuperado de http:// biblioteca.clacso.edu.ar/clacso/se/20160202103454/ Plurinacionalidad.pdf

unDP. Objetivos de Desarrollo del Milenio Informe 2015. Nueva York. Recuperado de http://www.un.org/es/millenniumgoals/pdf/2015/mdg-report-2015_spanish.pdf

unfCC. Aprobación del Acuerdo de París. Recuperado de http://unfccc.int/resource/docs/2015/cop21/spa/109s. pdf

Villada, J. (2016). Energías Renovables a nivel mundial y regional. Ecuador: INER.

White, B. (2012). Transacciones de tierras, desposesión y el futuro de la agricultura. CIP Ecosocial.

WWF. (2013). Comunicación presentada a SBSTA-Views on Framework for Various Approaches. Recuperado de http://unfccc.int/resource/docs/2013/ smsn/ngo/331.pdf 